\title{
Theologische Aussenansichten
}

Ralph Kunz

\section{Lob der Externität}

Wer einer Sache zu nahe steht, wer in sie involviert und verwickelt ist, hat keinen Überblick. Aus der Distanz betrachet, sieht man die Sache objektiver. Das ist der Vorteil der Externen. Weil sie unparteiisch und unvoreingenommen sind, können sich die Externen ganz der Sache zuwenden. Im Unterschied zur Innensicht eines Beteiligten, die auf Empfindungen, Erlebnisse und Erfahrungen gründet oder auf Bindungen Rücksicht nimmt, ist die Aussensicht eines Beobachters idealerweise objektiv. Beispiele für diese Einschätzung und Wertschätzung externer Urteilsfindung sind schnell zur Hand: Eine Unternehmensführung holt sich externe Berater, um Betriebsblindheit zu bekämpfen, die UNO schickt externe Beobachter in ein Krisengebiet, um Wahlbetrug zu verhindern oder eine Fakultät bestellt ein externes Gutachten, um im umstrittenen Fall eine dritte Stimme zu hören.

Die eigentliche Sachkompetenz der Externen hat freilich nichts mit ihrem Standort zu tun. Externität ist keine Garantie für Objektivität. Auch externe Berater können die Lage falsch einschätzen. Die unparteiische Stellungnahme oder unvoreingenommes Sicht ist in der Regel sachlich, der externe Standpunkt ist idealerweise objektiv. Was die Regel ist, muss nicht immer der Fall sein. Der Anspruch, objektiv an Wirklichkeit heranzutreten, ist nicht notwendig mit Externität verknüpft. Kritischer gesagt: Es gibt den externen Idealbeobachter nicht. Es gibt auf jeden Fall keine Wirklichkeit, die in der Weise unabhängig von Beobachtern existiert, dass ein Beobachter "von aussen" auf sie Bezug nehmen könnte. Wer etwas objektives über die Objektivität der Externen sagen will, geht also mit Vorteil auf Distanz zu übersteigerten Erwartungen.

\section{Religionswissenschaft als Aussensicht der Religion}

Die alltagssprachliche Verwendung der Aussen-Innen-Metaphorik, die dem Lob der Externität zugrunde liegt, schärft aber trotz oder gerade wegen ihrer Unschärfen ein Bewusstsein für die Grenzen der externer Objektivität, Grenzen, die im Blick auf Religion als Gegenstand der Forschung interessant sind. 
Fritz Stolz nennt in seiner Einführung in die Religionswissenschaft die Forderung eines objektiven Herantretens an eine Religion "pure Naivität«. Dennoch unterscheidet er zwischen einem Durchdenken der Religion "von innen" und "von aussen«. Religionswissenschaftler können ihren Gegenstand zwar nicht strikte "von aussen" erfassen, weil sie - wie alle Menschen - als Beobachter immer auch Beteiligte sind, aber sie können sich um "grösstmögliche methodische Distanz zum eigenen Standort" bemühen. Und weil dieser neue Standort ausserhalb des religiösen und innerhalb des wissenschaftlichen Wahrheitsverständnisses lokalisiert werden kann, handle es sich um eine Aussensicht der Religion. Was sich von diesem wissenschaftlichen Standort extern als religiöse Wirklichkeit entdecken lässt, erfassen und verstehen die Wissenschaftler freilich nur, insoweit sie sich selbst und ihr Verständnis der Wirklichkeit in dem Beobachteten verstehend wiederentdecken. Deshalb seien, sagt Stolz, alle religionswissenschaftlichen Untersuchungen "von begrenzter Reichweite". Sie tragen Fragen an den Gegenstand heran, die diesem fremd seien, und der Gegenstand hat immer Aspekte, welche sich der Interpretation des Forschers entziehen. ${ }^{1}$

Gerade im Fall der Religion liegt es auf der Hand, dass Bedarf für eine Wissenschaft gegeben ist, die sich im Bewusstsein ihrer begrenzten Reichweite kritisch und vernünftig um ein objektives Verständnis der Sache bemüht. Solange es Menschen gibt, die Religion praktizieren, ist die wissenschaftliche Beschäftigung mit der Bedeutung und Wirkung dieser Praxis von allgemeinem Interesse. Für bestimmte Zwecke lässt sich die Wirklichkeit der gelebten Religion - und das heisst auch ihre faszinierende Vielfalt, ihre Kraft und ihr Konfliktpotential - am besten aus einer gewissen Distanz beobachten und interpretieren. Der Erkenntnisgewinn, der von einer externen Betrachtung religiöser Phänomene erwartet werden kann, ist unbestritten. Es liegt auf der Hand, dass die damit verknüpfte Aufklärungsarbeit nicht zwingend von Menschen geleistet werden muss, die religiös sind. Wer wollte das bestreiten.

${ }^{1}$ Vgl. Fritz Stolz, Grundzüge der Religionswissenschaft, Göttingen 1997, 40. 


\section{Der Erfahrungsbezug der Theologie}

Bestritten wird aber das Postulat, dass die "grösstmögliche methodische Distanz zum eigenen Standort" eine Bedingung für Wissenschaftlichkeit sein soll. Eine konsequente Anwendung dieser Regel würde schnell zu problematischen Konsequenzen im Wissenschaftsbetrieb führen. Darf zum Beispiel ein Forscher, der die Geschichte des Gebets im 20. Jahrhundert untersucht, nicht beten? Anders gefragt: Wenn er es doch tut und beispielsweise Gott um Weisheit bittet, bevor er ein Buch über Euchologie liest, hat er dann die Regel der "grösstmöglichen Distanz" verletzt? Generell gefragt: Wann beeinträchtigen religiöse Praktiken die Qualität wissenschaftliches Schaffens? Was heisst in diesem Fall "Distanz zum Standort«?

Absolute Objektivität zu fordern, ist naiv. Ebenso naiv ist die Annahme, es gebe einen neutralen Grund, so etwas wie einen kulturlosen und religionsfreien Zugang zum religiösen Phänomen. Auch die "grösstmögliche methodische Distanz" verweist auf einen Standort innerhalb eines kulturellen Kontextes. Weder die Methoden noch der Gegenstand noch die Subjekte, die Methoden anwenden, um religiöse Gegenstände zu erforschen, lassen sich an einen externen Standort versetzen. Denn das sprachlich-kulturelle Symbolsystem Religion ist nur mit Methoden und von Subjekten als konkrete Praxis in einer konkreten Praxis erfassbar. Eine Beschreibung von Religion, die davon abstrahiert, beschreibt ein Phantom. Und Forscher, die meinen, sie können davon abstrahieren, von welchem Standort sie Wissenschaft treiben, haben eine naives methodologisches Bewusstsein. Für die Interpretation einer konkreten Religion ist deshalb das methodisch kontrollierte Nachdenken über den eigenen Standort und damit verbunden das Ineinander von Erfahrung und Reflexion konstitutiv.

Im Haus der Wissenschaft hat sich die Theologie als eine Form des Nachdenkens etabliert, in der sich Innensicht und Aussensicht der christlichen Religion perspektivisch verschränken. Das heisst: Theologie ist ein Nachdenken, das sich nicht auf unmittelbare Empfindungen und Erlebnisse bezieht. Der Erfahrungsbezug, der in der Theologie geltend gemacht wird, ist ein vermittelter. Theologen sind keine Schamanen, sie interpretieren die Tradition. Sie rufen nicht Geister, sondern vertrauen auf die Kraft des Geistes. Theologie ist zugleich ein Nachdenken, in dem Interpretationsarbeit als ein wesentlicher Teil der religiösen Erfahrung verstanden wird. Indem 
sie sich nicht auf eine grösstmögliche methodische Distanz zum eigenen Standort verpflichten lassen, wehren sich Theologen also gegen einen naiven Erfahrungsbezug. Aufgabe der Theologie ist es vielmehr, über die Voraussetzungen, Vollzüge und Folgen des Glaubens an Gott für das Leben der Menschen nachzudenken. Theologie ist die Reflexion einer Praxis, in der mit der Wirklichkeit Gottes auch und gerade im Geistigen - gerechnet wird.

Theologen fragen im Unterschied zu Religionswissenschaftlern nach dem, was Gott im Leben der Menschen wirkt. Die Wissenschaftlichkeit der Theologie zeigt sich daran, dass sie eine methodisches Instrumentarium zur Verfügung stellt, um die Geschichte und die Gegenwart des Glaubens kritisch zu erforschen. Wer Theologie treibt, ist insofern in Religion verwickelt und involviert, als er oder sie Kriterien ins Spiel zu bringen versucht, die christliche und andere Religionen kritisierbar machen.

\section{Ist Theologie eine Innensicht der Religion?}

Macht der Bezug auf die Geschichte und Gegenwart des Glaubens die Theologie zur Innensicht? Ist sie tatsächlich ein Durchdenken der Religion "von innen", wie das Stolz vorschlägt? Die meisten Theologen bestreiten dies vehement, weil "von innen" insinuiert, dass eine theologische Sicht der Glaubenspraxis christliche Religion nicht kritisieren kann. In den historischen Fächern wird argumentiert, dass die Erforschung biblischer Schriften und kirchengeschichtlicher Dokumente nach den gleichen methodischen Grundsätzen verfährt, wie sie die Religionsgeschichte zur Anwendung bringt. Historische Kritik arbeitet agnostisch. Es geht nicht darum, die Glaubenswahrheit "von innen« gegen Angriffe "von aussen« zu verteidigen. Die Befragung der Glaubensgeschichte unter historischer Perspektive ist eine notwendige Voraussetzung für die theologische Kritik der christlichen Religion. Ohne historische Relativierung wäre das wissenschaftliche Gespräch über die Praxis des Glaubens nicht möglich. Nur zielt sie, wenn sie sich auf die Praxis des Glaubens bezieht, über ein rein historische Kritik der Religion hinaus. Denn das Interesse der historisch orientierten kritischen Theologie ist es, fundamentalistische, biblizistische, fideistische und dogmatistische Verwirrungen des Glaubens zu vermeiden.

Auch die Aufgabe der systematischen und praktischen Theologie wird mit der Ortsangabe »von innen« verzeichnet. Wenn Glauben 
etwas mit Gottes Gegenwart in der Welt zu tun hat, ist das Nachdenken darüber keine Innensicht des menschliches Leben. Wie könnte sonst von Gott als einer Realität die Rede sein, die vom Glauben unterschieden werden kann? Natürlich macht die Unterscheidung von Glaubensgrund und Glaubensvollzug nur dann Sinn, wenn auch ein Sinn darin gesehen wird, Gott und Menschen zu unterscheiden. Die Annahme, dass diese Unterscheidung sinnvoll ist und dass sich aufgrund des Evangeliums menschliches Leben sinnvoll gestalten lässt, ist aber nicht zwingend eine interne Sicht der Praxis.

Für den christlichen Glauben ist die Anerkennung Gottes keine theoretische Angelegenheit, sondern verbunden mit einer bestimmten Lebensweise, in der Bezug auf Gott genommen wird. Gott anerkennen heisst sich selbst im Verhältnis zu Gott erkennen. Glaubenspraxis und Wissenschaft nehmen den Bezug auf Gott unterschiedlich wahr. Theologisch denken heisst, streng darauf zu achten, dass Gott sich zum menschlichen Leben - notabene auch zur Religion - ins Verhältnis setzen lässt. Dass Menschen sich Gott gegenüber verhalten, ist kein zwingender Gedanke. Theologie kann und muss Gott nicht verteidigen. Aber Theologen sollen im Gespräch mit anderen Wissenschaften die Argumente für den möglichen Sinn ihrer Gedanken so präsentieren, dass sie rational nachvollziehbar sind. Glaube wird reflektiert und diskutiert, einfordern kann man Glauben im wissenschaftlichen Diskurs nicht.

\section{Begrenzte Reichweiten}

Theologische Aussagen sind von begrenzter Reichweite, gerade weil sie eine Aussensicht behaupten, die mit einem Wahrheitsanspruch verknüpft ist. Wenn alle Versuche, über Religion etwas Objektives zu sagen, letztlich durch die Wahrheit begrenzt sind, empfiehlt es sich, die unterschiedlichen Herangehensweisen von Theologie und Religionswissenschaft nicht mit einer Innen-Aussen-Unterscheidung zu definieren. Die naive Verwendung der Metaphorik nährt das Missverständnis, Theologie sei befangen und gefangen in einer religiösen Sichtweise oder Weltanschaung und daher keine eigentliche Wissenschaft. Will man diesen Fehlschluss vermeiden, ist man genötigt, sich vom Wahrheitsversprechen einer übertriebenen Wissenschaftlichkeit zu distanzieren. Gleichzeitig würde man Einsichten in Wahrheiten verspielen, wenn man dem Reden von sinnen ssen k keine erkenntnissteigernde Wirkung zutraut. Wer sich in der 
Wissenschaft von einer übertriebenen Wissenschaftlichkeit distanzieren will, setzt ja voraus, dass man sich zur Wissenschaft verhalten kann, ohne sich von ihr zu verabschieden. Wie ist es aber möglich, übertriebene Externität zu kritisieren, ohne sich dabei als externer Besserwisser in genau die Widersprüche zu verwickeln, die man eigentlich kritisieren möchte? Das Innen im Aussen und das Aussen im Innen geraten aneinander und durcheinander, je nach dem von welchem Standpunkt aus man welchen Blickrichtung wählt. Es gibt immer ein Äusseres zu einem Aussen. Das Spiel lässt sich bis zum Äussersten treiben, um dort wieder im Innersten zu beginnen. Ein Spiel ohne Grenzen und gerade dadurch begrenzt.

Grenzen sind interessant. Es ist besonders interessant, den Grenzen der Externität in Bezug auf Religion auf die Spur zu kommen. Weshalb ist es naiv, von einer objektiven Religionswissenschaft $\mathrm{zu}$ sprechen? »Reine« Externität, so lautete eine Antwort, gibt es nicht, weil jedes Innen und Aussen an ein Subjekt gebunden ist, das nicht aus seiner Haut schlüpfen kann. Man müsste, so könnte man den Gedanken fortsetzen, schon Gott sein, um die Dinge ganz objektiv zu sehen. In einer unbegrenzten, freien und unabhängigen Position, interessiert und doch nicht in Interessen verwickelt, wohlwollend aber nicht korrumpierbar, könnte man der Sache, die man untersucht, letztlich gerecht werden. Das ist naiv geredet. Aber um das Ausloten dieser Naivität geht es. Wissenschaftler möchten wissen, wie es sich mit dem, was der Fall ist, tatsächlich verhält. Sie möchten gleichsam ins Innerste der Dinge sehen, wissen, dass sie es nicht können und versuchen es trotzdem. Wenn sie wissenschaftlich arbeiten, müssen sie beides, ihr Wissen-Wollen und ihr Nicht-allesWissen-Können, vereinen. Wissenschaftler, die nachdenken, könnten zum Schluss kommen, dass sie mit dem Gedanken spielen, allwissend wie Gott zu sein. Dass sie mit diesem grösstmöglichen Gedanken spielen, hat Methode. Menschen, die spielen, stossen an ihre Grenzen. Und einige, die die Grenzen ihrer Spielmöglichkeiten austesten, stossen auf den Gedanken Gottes.

Theologie ist eine Wissenschaft, die das Thema der Grenzen traktiert, indem sie ihre Begrenztheit, die Grenzen der wissenschaftlichen Beschäftigung mit Gott, zum Thema macht. Kein Mensch kann wissen, wie sich die Sache mit Gott tatsächlich verhält. Man müsste Gott sein, um in dieser Frage zu einer Innensicht zu gelangen. Wenn aber Theologen in Gedanken Gott spielen wollen, 
könnten sie die Differenz zwischen dem, was sie verstehen wollen und dem, was sich in ihrem Verstehen abspielt, nicht mehr behaupten. Mit Karl Barths Paradox gesagt: "Wir sollen als Theologen von Gott reden. Wir sind aber Menschen und können als solche nicht von Gott reden. Wir sollen Beides, unser sollen und unser NichtKönnen, wissen und eben damit Gott die Ehre geben. ${ }^{2}$

\section{Epilog}

Theologen spielen mit dem Wort \Gott aufgrund von Gottes Wort. Von einem Wort Gottes zu reden, impliziert, dass Gott nicht nur als externe Begrenzung menschlichen Wissens vorgestellt wird. Dass Gott spricht, setzt seiner Unbegrenztheit Grenzen. Es sind Grenzen, die er sich selbst setzt. Gott ist wahrhaftig Mensch geworden - eine Grenzüberschreitung von der Externität in die Interna der Welt.

\footnotetext{
${ }^{2}$ Karl Barth, Das Wort Gottes als Aufgabe der Theologie (1922), in: Albrecht Beutel et al. (Hg.), Homiletisches Lesebuch, Tübingen 1986, 43.
} 